\author{
Military Technical College \\ Kobry El-Kobbah, \\ Cairo, Egypt
}

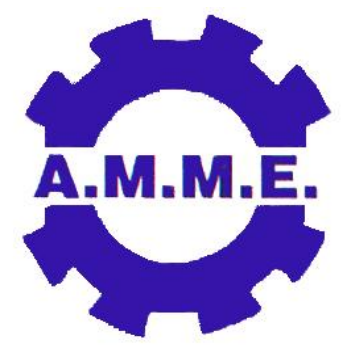

14th International Conference on Applied Mechanics and Mechanical Engineering.

\title{
Computer Aided Engineering (CAE) Techniques Applied to Hip Implant
}

\author{
By \\ M. E. Abo-Elnor * \\ M. S. Abo_Elkhair* \\ A. E. Radi **
}

\begin{abstract}
:
Methods to predict contact stresses in femoral prostheses can provide an improved understanding of load distribution. The objectives of this study is to apply advanced computer aided engineering techniques (CAE) for predicting stress transfer to femur after implantation of standard and short femoral stems (Proxima stem). A perspective threedimensional geometry model for the femoral bone using subject-specific geometry from $X$ ray computed tomography image data $(C T I)$ is used to create $3 D$ CAD model. Forces acting on the femur in different gait regimes were reviewed and compared while loaded with standard and then with Proxima stem. And finally a developed finite element analysis model based on the created CAD model with forces acting on both femurs loaded with standard and Proxima stem is analyzed.
\end{abstract}

\section{Keywords:}

Computer aided engineering, Biomechanics, Femur modeling, implant 


\begin{tabular}{ll}
\hline$*$ & Egyptian Armed Forces \\
$* *$ & Professor of orthopedics, Faculty of medicine Ain-Shams Univ. Cairo, Egypt
\end{tabular}

\section{Introduction:}

Anatomic short femoral prostheses with a prominent lateral flare have the potential to reduce stress-shielding in the femur through a more physiological stress distribution to the proximal femur. Femur bone crack and fracture is one of the most common forms of injuries during accidents. Body weight is transferred through pelvis to femur by hip joint. Bone is a living tissue, which continuously rebuilds its structure according to the direction of loads exerted on it. After insertion of a metal prosthesis into the medullary canal, the load equilibrium and the remodel tissues in the bone are disturbed. Stress-shielding activity of the implant (Fig. 1), which is stiffer, makes the bone atrophies [1]. Reduction of stresses in bone with respect to the natural state causes its adaptation to the new conditions manifested by mass changing (external remodeling) or bone density changing (internal remodeling).

The latter is especially dangerous because, as already indicated, it can cause aseptic loosening of the implant. Studying different factors affecting stress-shielding activity required appropriate modelling and simulation of the hip stem and femoral bone along with cemented media used in hip replacement surgery.
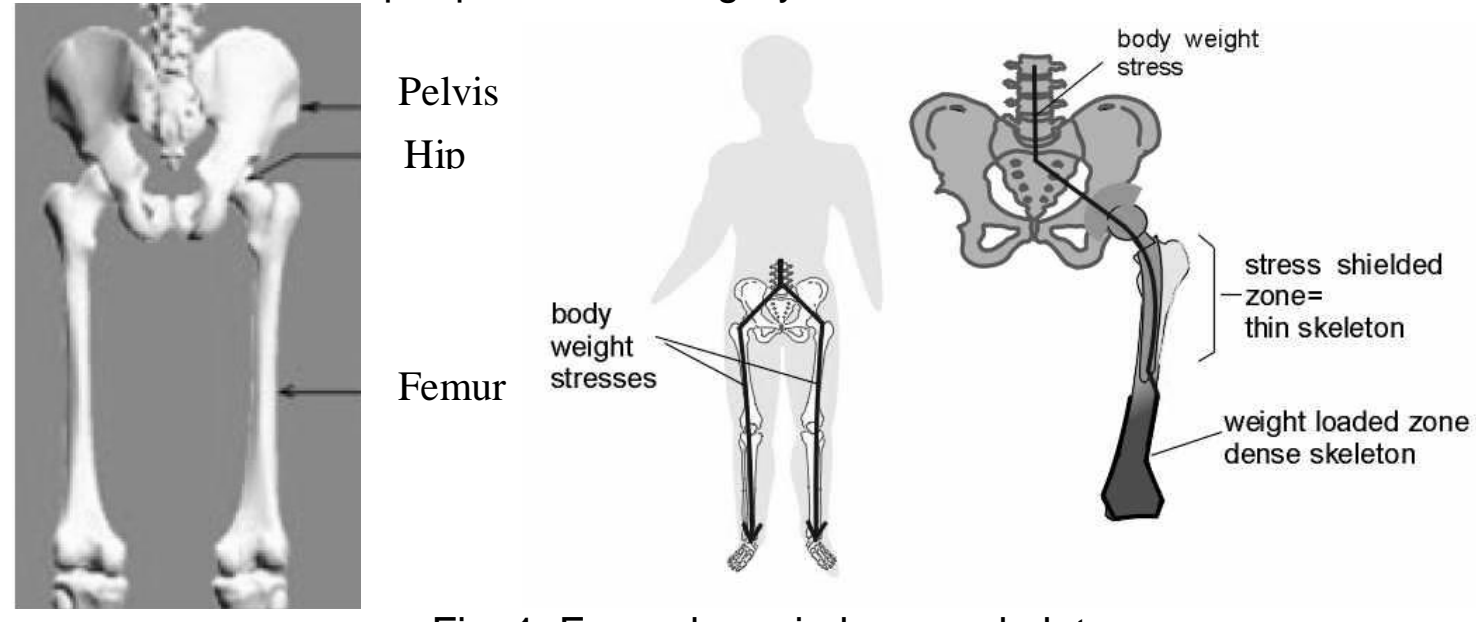

Fig. 1, Femur bone in human skeleton

The proper modeling of femur hip replacement can be achieved through the following processes:

1- Acquisition of computer tomography (CT) data of the femur.

2- Geometrical modeling of the femur and design of prosthesis stem in a computer-aided design (CAD) system.

3- Calculation of forces acting on hip during different gait regimes.

4- Finite element analysis of the selected implant prosthesis and bones.

\section{Materials and Methods}

\section{Acquisition and processing of CT data}

Novel techniques have been developed to convert 3D image data, obtained from Computer Tomography, automatically into numerical meshes suitable for Finite Element 
(FE) analysis. CT measurements [2] of the diseased hip joint were performed in order to acquire coordinates of the points defining the femur shape. The CT images obtained were then processed to filter the required data and detect the femur shape (Fig. 2).
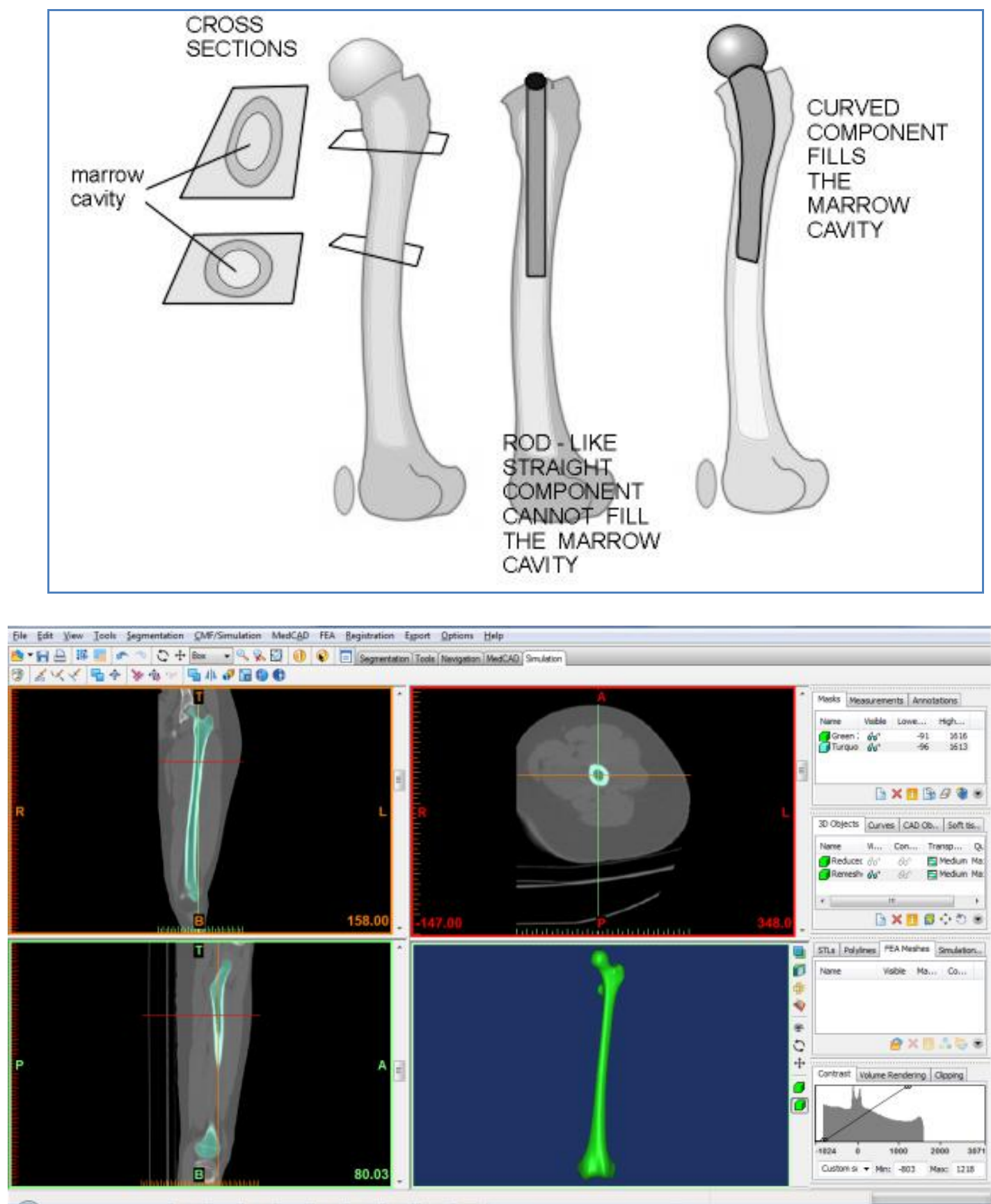

Fig. 2, CT images implementation to create 3D model of femur bone

Mimics is a software suite that performs the segmentation of the anatomy through sophisticated three-dimensional selection and editing tools [3]. The Mimics software is an image-processing package with $3 \mathrm{D}$ visualization functions that interfaces with all common scanner formats.

Femur Geometrical modeling and prosthesis stem design The processed CT data obtained by Mimics is then used either as a meshed model for rapid Finite Element Analysis of the femur or to create a 3D cad model, using AutoDesk 
Inventor CAD software, for prosthesis stem design as shown in figure (3).

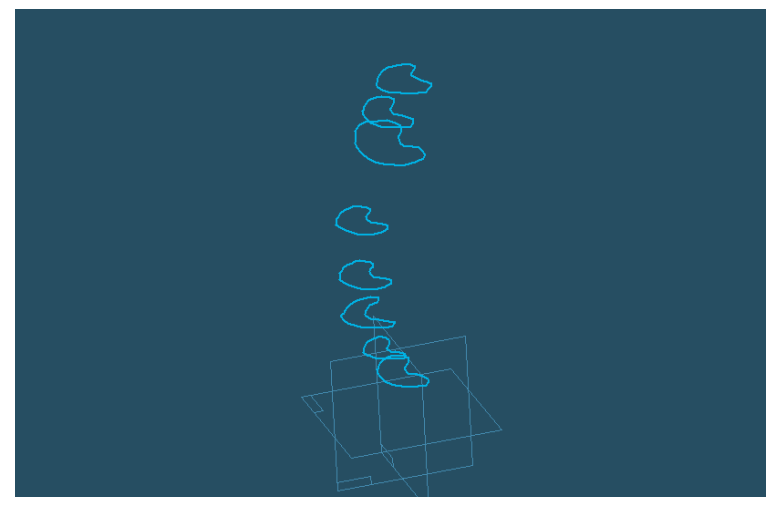

Slices for further CAD uses

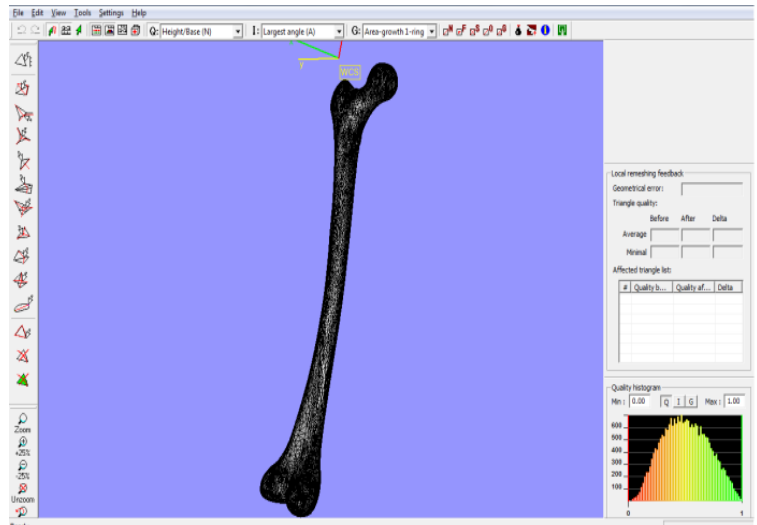

Mesh model for Finite Element Analysis

Fig. 3, 2D slices and 3D mesh results of the processed CT data

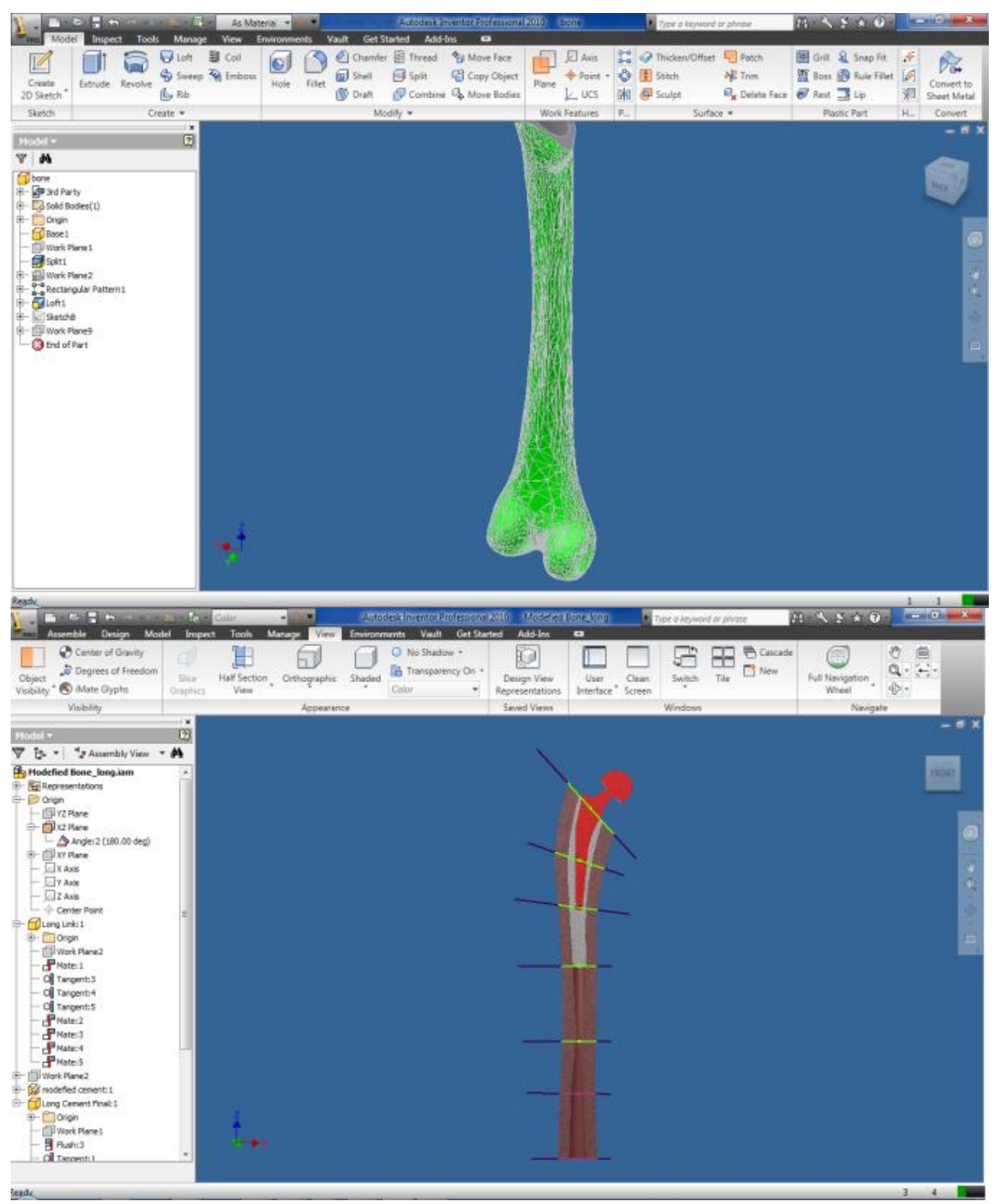


Fig. 4, Processing of the 3D model (cutting) in order to obtain the interested part of the femur

The proposed custom implant design was done using Autodesk Inventor CAD software (Fig. 4). A CAD model of the patient's distal femur was imported and used as the base for the design. The articulating surface was kept intact to avoid altering the gait and to preserve the correct patellar grove so as to prevent resurfacing and patellar dislocation.

A set of spline curves were created along the interface surface in a radial pattern (Fig. 5), and a single spline was created to connect all curves in a central plane. A swept-blend command was used to create the smooth articulating surface. The bone-implant interface was created using a new set of curves in the same planes as the original curves. The inner curves were offset from the original curves and then manually edited to avoid any undercuts. The offset distance was kept uniform to create an implant with constant wall thickness. A new center plane curve was created as well to prevent the need for undercuts. A swept-blend-cut command was used to create the bone-implant interface. Additional cuts and fillets were added to provide an implant with smooth surfaces and edges.

\section{Forces acting on hip during different gait regimes}

Muscle forces exerted during human movement provide insight in tissue load and muscle function and/or malfunction. The use of musculoskeletal models in the estimation of muscle force has been widely reported in the literature [4].

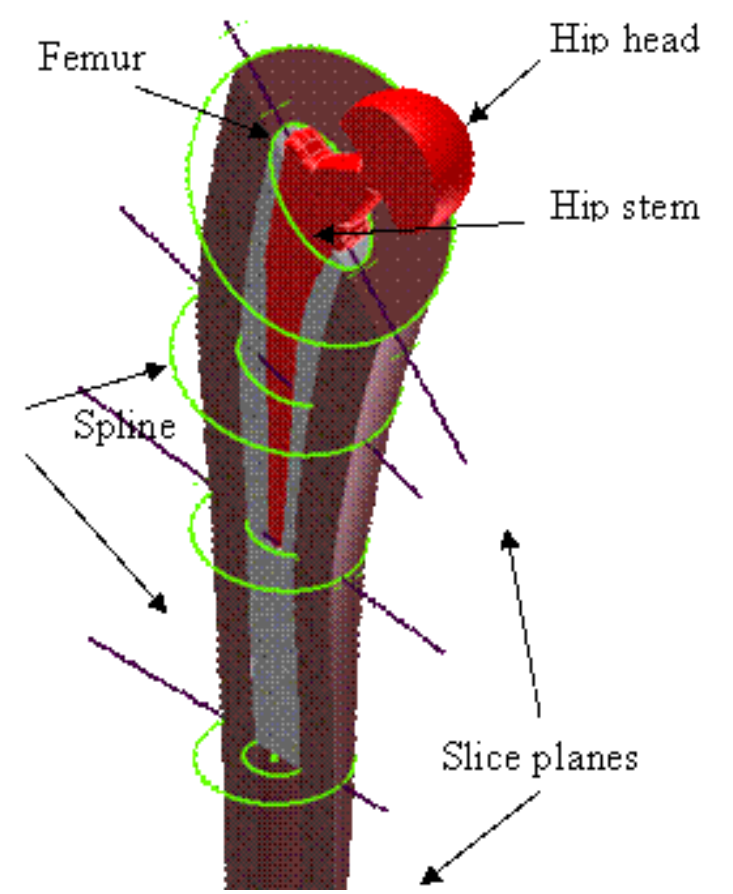

Fig. 5, Femur bone and prosthesis stem modelling 


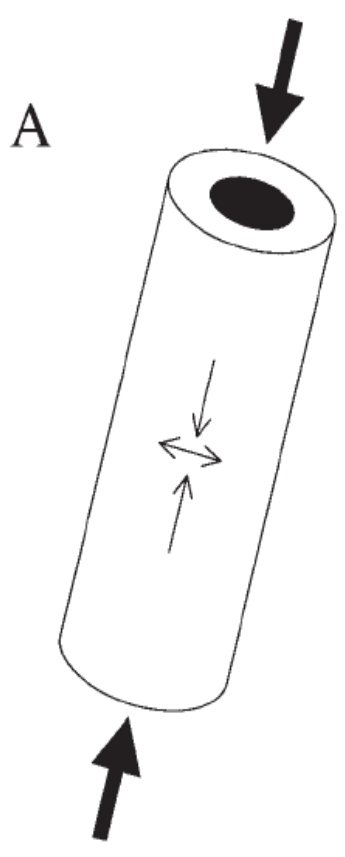

Axial compression

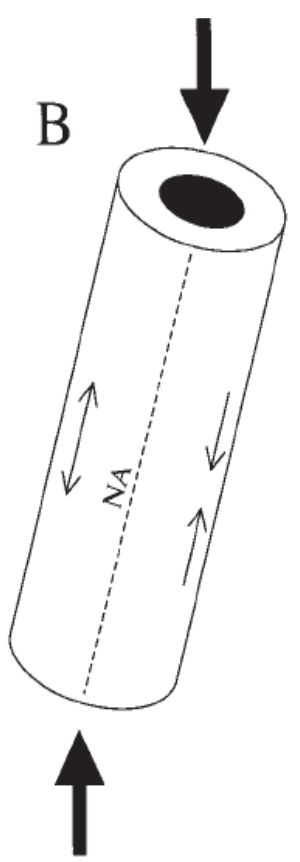

Bending

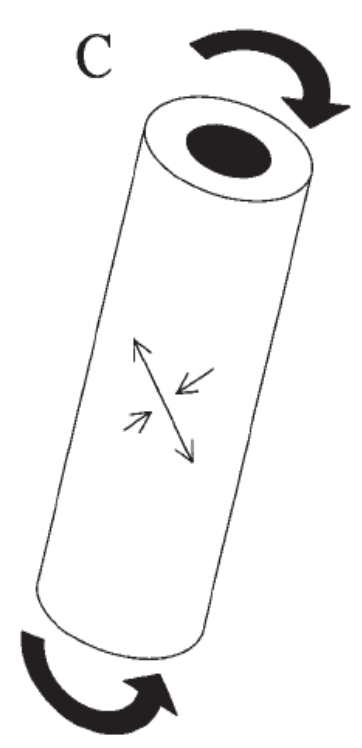

Twisting

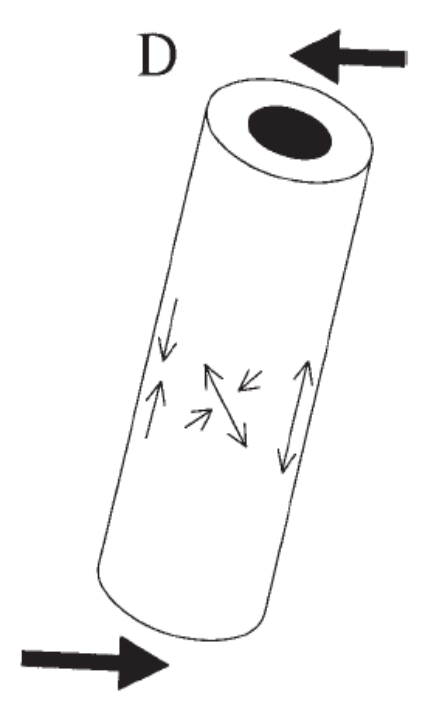

Shear

Fig. 6, Beam and cross-section views of applied forces and strain distributions in compression (A), bending (B), twisting (C), and shearing (D).

bones are loaded in four possible ways [5], depicted in figure (6), they can be compressed axially, bent, sheared, and twisted. Often these loads occur in combination (most long bones are mostly bent, but also compressed and twisted to varying extents).

Based on terminologies described in figure (7), a literature review of several analytical and experimental results [6] are plotted in figure (8) and figure (9). These results are used to simulate forces acting on femur hip for the finite element analysis.

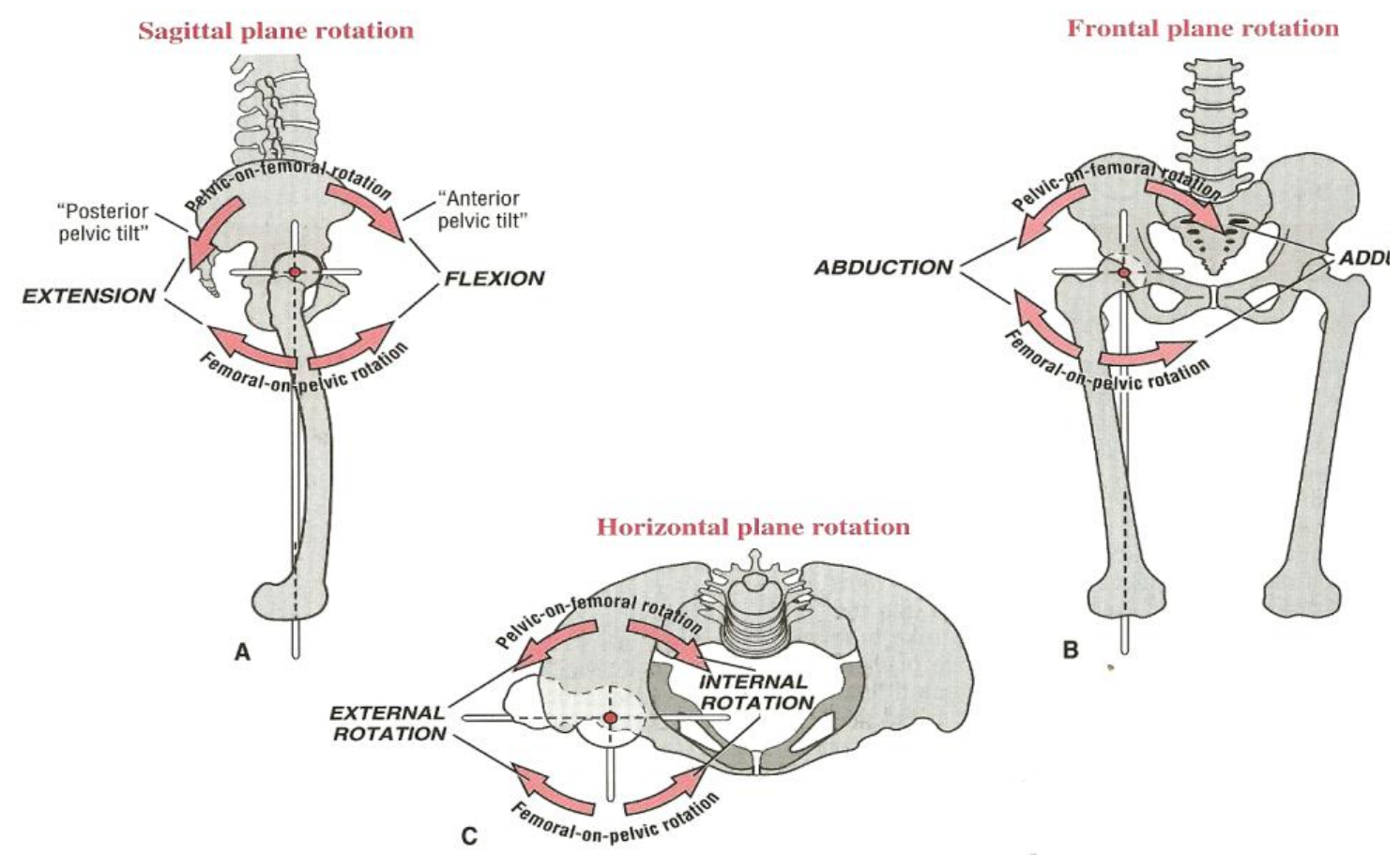

Fig. 7, femur degree of freedom terminologey 

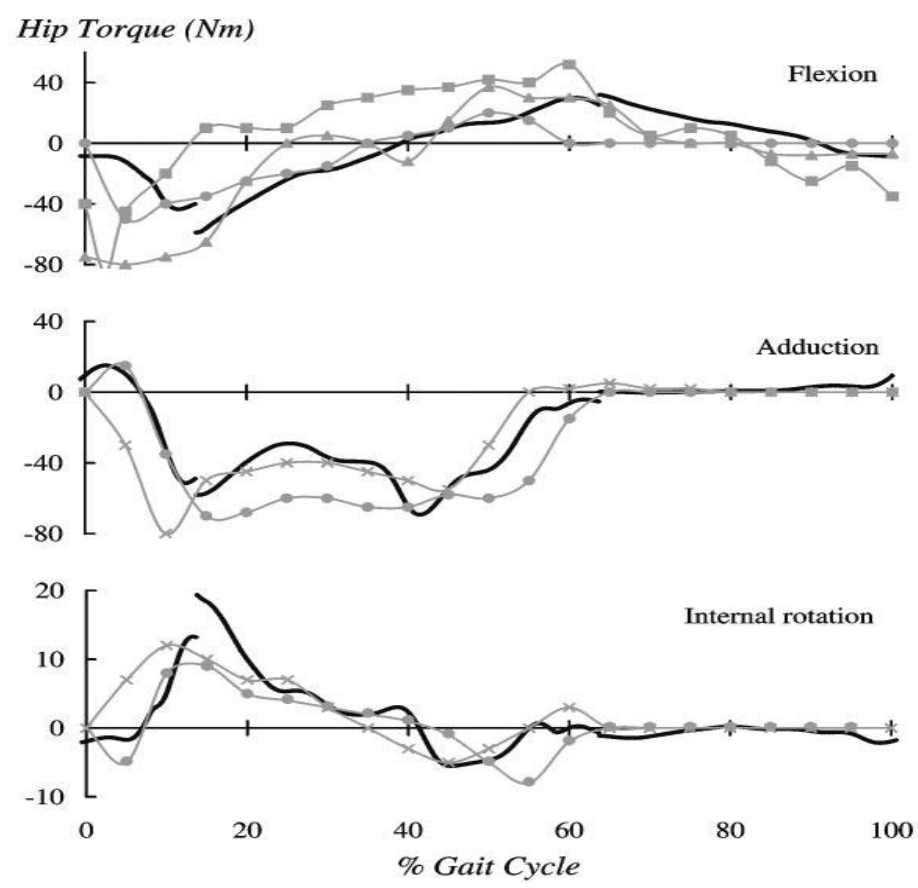

Fig. 8, The net muscle moments applied about the axes of the hip joint Hip Contact Force / BW

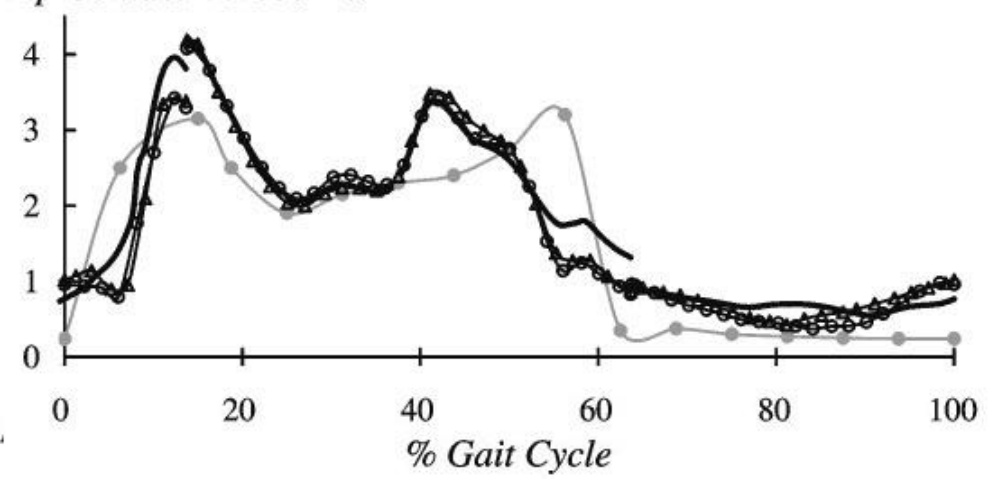

Fig. 9, Joint contact force in the hip

\section{Finite element analysis}

A finite element model of spacer and femur was developed to analyze stresses [7]. The analysis was performed using ANSYS, a FEA software (ANSYS Inc., Canonsburg, United States). CAD model was obtained as described before and transferred to ANSYS for FEA. Figure (10) shows both boundary condition and mesh distribution. Effect of knee-femur connection is modeled as fixed boundary for simplicity. The cancellous bone within the femur head has a Young's modulus of $1300 \mathrm{MPa}$ and Poisson's ratio 0.28 while bone cement were analysed with a Young's modulus of $2200 \mathrm{MPa}$. A $4 \mathrm{kN}$ load was applied to the femoral head. 


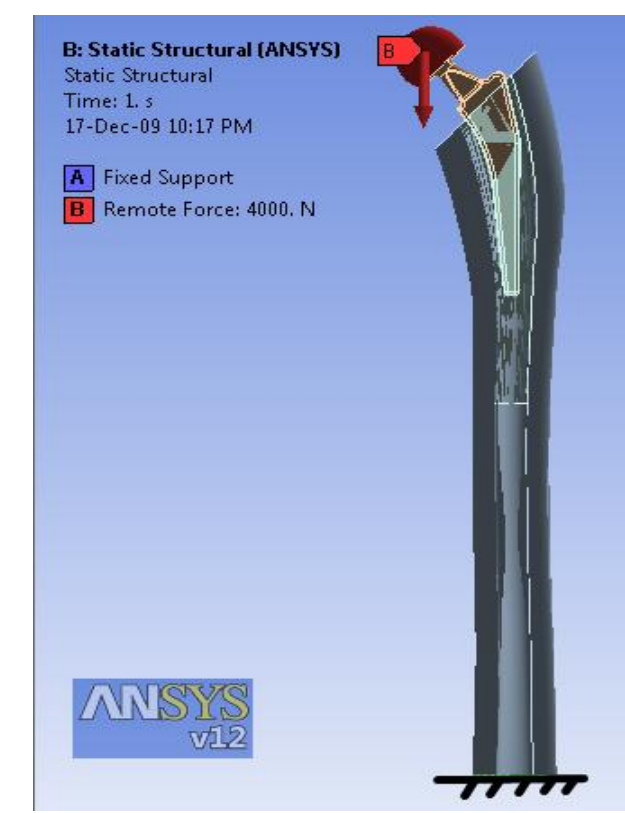

femur force and boundary condition

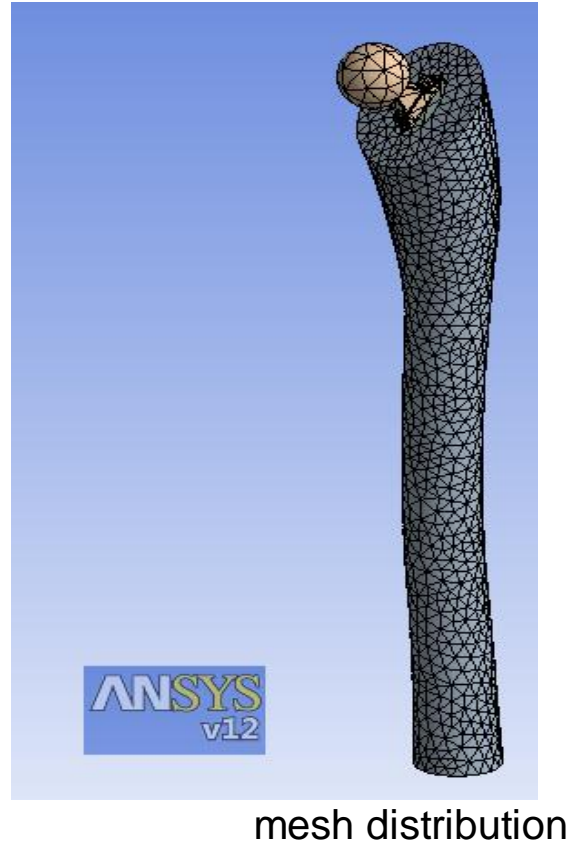

mesh distribution

Fig. 10, Initial and boundary conditions for the FEA

\section{Results and Discussion}

Finite element results reveal the capability of the proposed computer aided design process to handle such an analysis requirements for orthopedic implants evaluation. As shown in figure (11) and figure (12), the homogenous distribution of the strain and stress fields across the contact layer between hip stem and cemented material and between cemented material and femur bone is an accepted proof of contact establishment of the cemented fill and both hip and femur. Extensive analysis of hip stem design, material and effect of using cement or cement-less installation of the hip stem along with studying the effect of cement material can be carried out using proposed model. Also the effect of different loads in gait regimes on stress-shielded zone will be analyzed latter on.
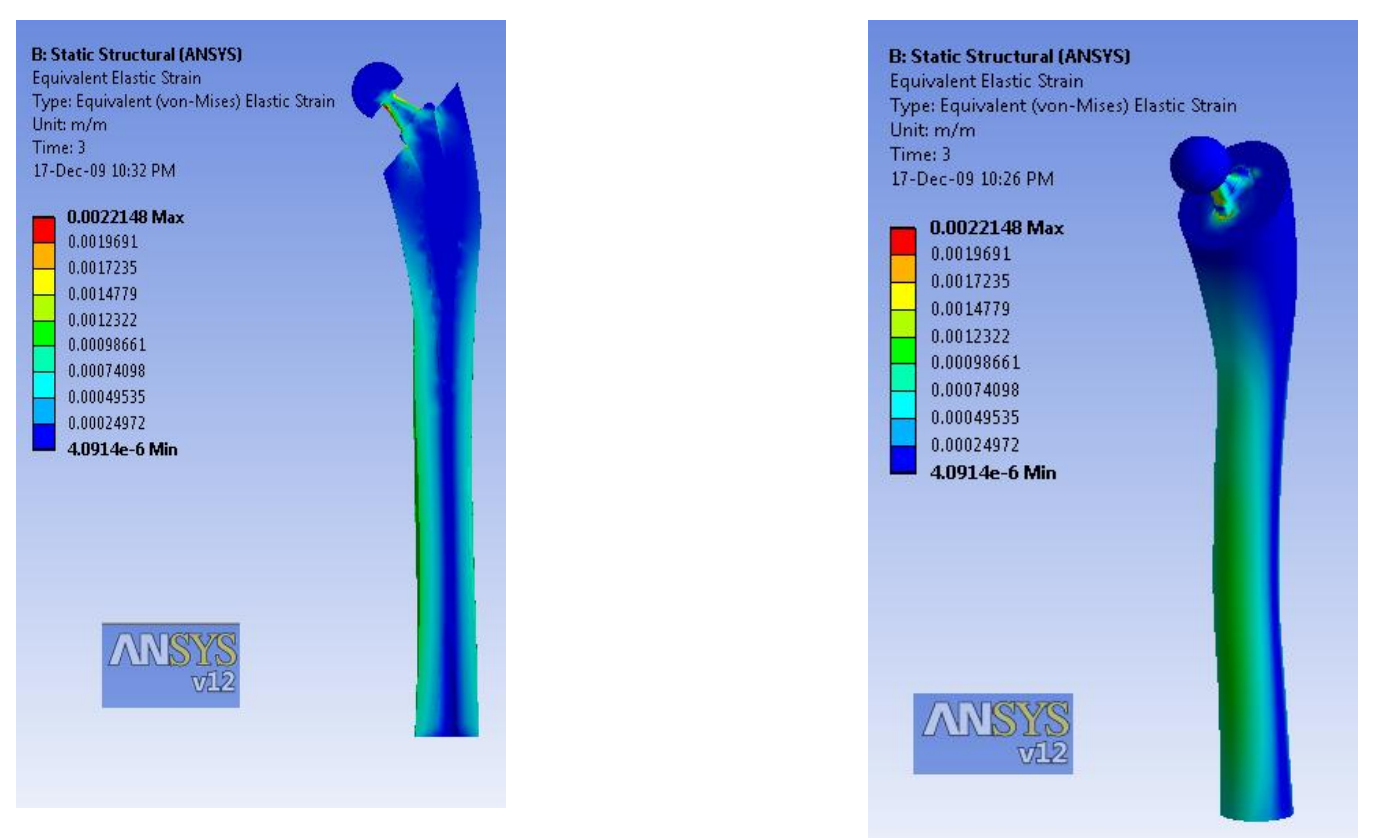

Fig. 11, Elastic strain distribution in section view (left) and 3D view (right) 

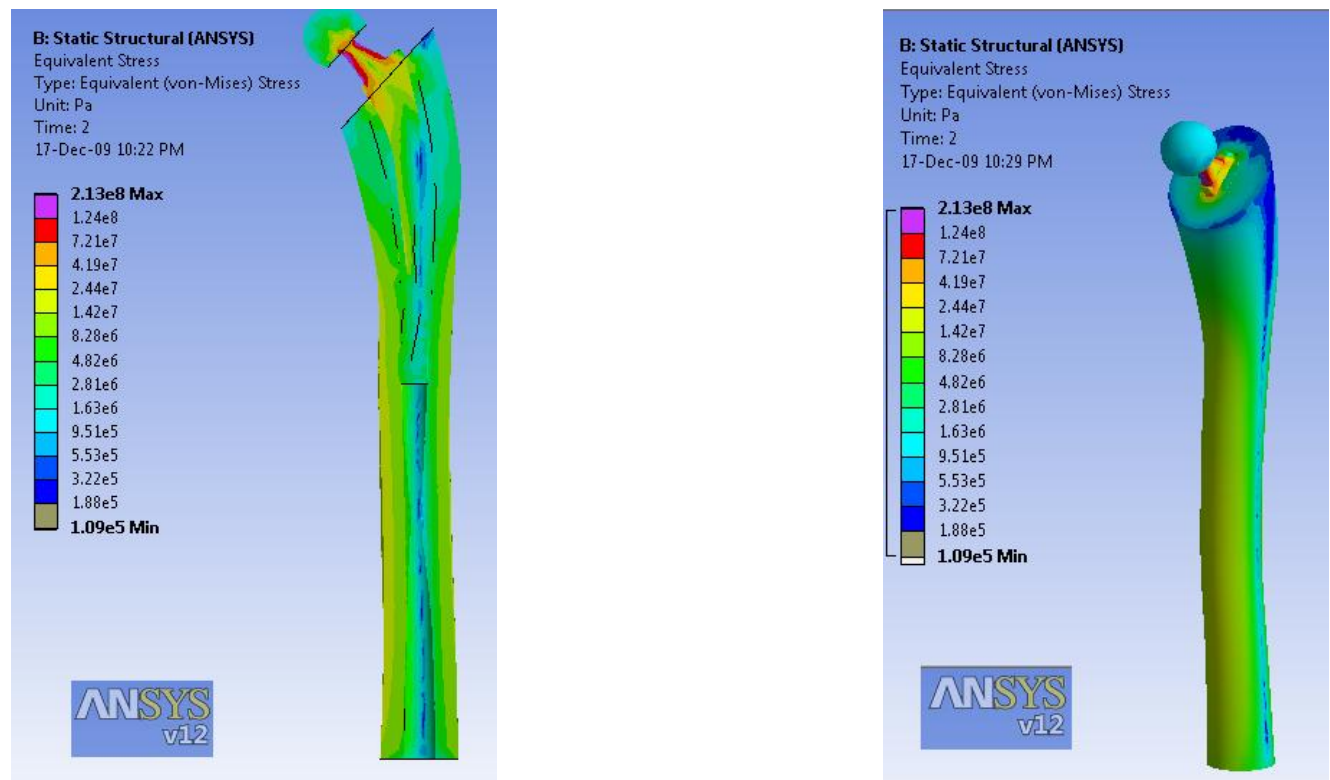

Fig. 12, (von-Mises) Stress distribution in section view (left) and 3D view (right)

\section{Conclusions}

Rapid custom modeling of human skeleton using advanced computed tomography (CT) techniques is believed to be the most effective way of selection of the right implant design for surgery. Advanced computer aided engineering (CAE) techniques such as CAD and FEA can provide better examination and analysis of the designed implant before surgery operation to insure high durability of the designed implant. In the research field, factors affecting stress distribution to human bones via the implant can be done using the CAE facilities for implant design optimization.

\section{References:}

[1] Mihai Ovidiu GHIBA, Lucian RUSU, "Geometrical design of custom-made femoral stem prostheses", Fascicle of Management and Technological Engineering, Vol. VII, No. XVII, 2008.

[2] MIMICS (10.0) tutorial.

[3] K. L. Chelule, Dr. T. Coole, D.G. Cheshire, "Fabrication of medical models from scan data via rapid prototyping techniques"

[4] Erdemir, A., McLean, "Model-based estimation of muscle forces exerted during movements", Clinical Biomechanics Vol. 22, PP. 131-54, 2007.

[5] Osbjorn M. Pearson, Daniel E. Lieberman, "The Aging of Wolff's "Law": Ontogeny and Responses to Mechanical Loading in Cortical Bone", YEARBOOK OF PHYSICAL ANTHROPOLOGY, Vol. 47, PP. 63-99, 2004.

[6] Frank C. Anderson, Marcus G. Pandy, " Static and dynamic optimization solutions for gait are practically equivalent", Journal of Biomechanics, Vol. 34, PP. 153-161, 2001.

[7] T. Thielen, S. Maas, A. Zuerbes, et al, "Mechanical behaviour of standardized endoskeleton-including hip spacers implanted into composite femurs", International Journal of Medical Sciences, Vol. 6, No.5, PP.280-286, 2009. 